\title{
La evaluación de las prácticas de laboratorio mediante el uso de uve heurísticas y matrices de valoración
}

The evaluation of the practices of laboratory by means of the use of heuristic and first uve of valuation

\author{
Nelson Eduardo Alzate Betancur', María Angélica León ${ }^{2}$, Yessica Martín Bautista \\ y Leidy Gabriela Ariza Ariza ${ }^{4}$
}

1,2,3 Estudaintes de Licenciatura en Química. Universidad Pedagógica Nacional. ${ }^{4}$ Docente del departamento de Química. Universidad Pedagógica Nacional. dqu615 nalzate@pedagogica.edu.com dqu641 mleon@pedagogica.edu.co dqu643 ymartin@pedagogica.edu.co,lariza@pedagogica.edu.co

\section{Resumen}

La presente propuesta esta enfocada a realizar una reflexión del uso de las prácticas de laboratorio en el proceso de enseñanza y aprendizaje de la química en profesores en formación inicial. Para que estas permitan que el conocimiento sea asimilado e interiorizado por el estudiante de manera mas concreta. Por ello la necesidad de manejar diversidad de instrumentos que ofrezcan alternativas al momento de evaluar y sobre todo de aprender a través de herramientas metacognitivas como las UVE-heurística, la cual permite valorar todos los procesos hechos antes, durante y después de la práctica de laboratorio. Este conjunto de procesos hacen necesario el manejo de una matriz de valoración como instrumento que relaciona e incluye lo que espera el profesor, lo que debe desarrollar el estudiante, como un previo acuerdo al inicio del proceso.

\section{Palabras claves}

Enseñanza de la química, practicas de laboratorio, herramientas metacognitivas, evaluación, UVE-heurística, matrices de valoración, educación científica, actitudes científicas, alfabetización cientúfica.

\section{Abstract}

The present proposal this focused to make a reflection of the use of the laboratory practices in the education process and learning of chemistry in professors in initial formation. So that these allow that the knowledge is assimilated by the student of concrete way but. For that reason the necessity to handle diversity of instruments that offer alternatives at the time of to evaluate and mainly to leam through metacognitivas tools like the UVE-heuristic one, which allows to value all the processes done before, during and after the laboratory practice. This set of processes makes the handling necessary of a matrix of valuation like instrument that relates and indudes what waits for the professor, which must develop the student, like a previous agreement at the beginning of the process.

\section{Keywords}

Education of chemistry, you practice of metacognitivs, evaluation, UVE-heuristic, first laboratory, tools of valuation, scientific education, scientific attitudes, scientific alphabetization.

\section{Introducción}

Con el objeto de reflexionar sobre la enseñanza de la química en la educación superior y sus prácticas de laboratorio, este artículo se enfatiza en la utilización de la Uve Heunística de Gowin (1981), la cual se puede utilizar como estrategia de análisis tanto de la construcción (conocimientos 
científicos) como de la reconstrucción (aprendizaje) de los mismos. Esta técnica heurística fue desarrollada para ayudar a profesores y estudiantes a comprender la naturaleza y producción de conocimientos, a través de los registros en ella se pueden construir hechos que sean repetibles, fiables y consistentes, por ello es necesario tener ideas, teorías, estructuras conceptuales, entre otros. Los diagramas uve permiten la representación que puede hacer el estudiante para expresar cómo se construye algún fenómeno o problema. Esto incrementa la seguridad acerca de cómo y por qué es valioso la evaluación por este método, y como las matrices pueden conducir el trabajo que aquí se presenta.

La dinámica de enseñanza-aprendizaje que se lleva a cabo en las instituciones educativas debe generar espacios donde se fortalezcan las estrategias de formación desde diversos aspectos, en este caso especifico de las prácticas de laboratorio desde la alfabetización cient́fica "Hoy más que nunca es necesario fomentar y difundir la alfabetización cient́fica en todas las culturas y en todos los sectores de la sociedad,... a fin de mejorar la participación de los ciudadanos en la adopción de decisiones relativas a las aplicaciones de los nuevos conocimientos" Dedaración de Budapest, 1999 (citado de Gil, 2001), por tanto, los profesores no pueden ser tan solo transmisores del conocimiento sino generadores del mismo orientados desde la proposición y transposición de los conocimiento cient́ficos a los conocimientos cotidianos para que estos estén al nivel conceptual, procedimental y actitudinal de los estudiantes. En la educación cient́fica las prácticas de laboratorio se convierten en esenciales para aplicar los elementos cognitivos y procedimentales de lo que se aprende, ya que ellos pueden participar en la dinámica del conocimiento, especialmente en grupo, el profesor es el orientador no el "dictador" del proceso. Sin embargo, la realidad muestra que las prácticas de laboratorio en la educación que imparten muchos docentes, tienen poco peso, respecto a las dases magistrales.

Teniendo en cuenta el apartado anterior, es de vital importancia reconocer que las prácticas de laboratorio no solo deben hacer parte del ámbito educativo medio o superior, por el contrario la implementación desde la escuela primaria puede desarrollar habilidades que le permitirán al estudiante descubrir fenómenos que ocurren a diario y se reflejan en el entorno en el cual se desenvuelve. Las dases de prácticas que se podrían generar en la escuela estarían enfocadas a la indagación, exploración y observación, en las cuales ellos pueden inferir las causas, sin ver la necesidad de la operatividad a través de cálculos ni formulas como se hace a nivel superior.

Como lo propone Hodson (1994), el trabajo práctico en el laboratorio puede ser motivante, por medio de estímulos de interés y "diversión", al mismo tiempo que enseña técnicas fundamentales de laboratorio intensificando el aprendizaje de los conocimientos cient́ficos que desarrollan "actitudes cientúficas". Por esto, la ayuda del profesor debe ser suficiente para que el estudiante vaya pensando en lo que puede hacer y el significado de lo que hace en cada momento de la experiencia. El estudiante debe percibir la práctica como un pequeño trabajo de investigación (Solaz, 1990) por lo que una vez terminada se plasme no solo lo obtenido, sino lo analizado e interpretado, y como se podría aplicar a situaciones cotidianas especificas.

En la sociedad continuamente se destaca la influencia del lenguaje científico y de su impacto a nivel socioeconómico, pero ¿Cómo estos son manejados en el sistema educativo?, es importante tener en cuenta que la educación esta cambiando y que necesita innovación, consecuente con esto tiene valor directo la educación científica en la vida de cada individuo, dejando a un lado la imagen de ciencia limitada y descubridora (Chalmers, 1976). Bajo estas ideas, es importante reconocer el papel de la Ciencia, tecnología y Sociedad la cual nos impulsa la adquisición de una "cultura cientúfica" utilizando la alfabetización cientúfica como medio de incursión a una ciencia no elite (cientúficos asociales encerrados en el laboratorio), sino a una ciencia que sin ser tergiversada ni empobrecida conceptualmente, pueda ser adquirida y relacionada con diferentes contextos sociales. Para llevar a cabo esto en el aula es primordial, desarrollar criterios de evaluación que den un verdadero juicio no solo aualitativo sino cuantitativo del desarrollo que ante la critica social de la 
ciencias esta adquiriendo el estudiante, y al mismo tiempo qué aportes esta haciendo a la misma desde su observación en campos cotidianos.

Con respecto a lo fundamentado anteriormente, las prácticas de laboratorio que se diseñan y desarrollan en la enseñanza y aprendizaje de la química son la aplicación de interrelacionar los conocimientos (no solo adquiridos en el aula), los contextos (que no se limitan a aulas especializadas, sino a lugares apropiados), las capacidades y las actitudes hacia la ciencia. Todo este proceso se realizara con el fin de incorporar una nueva forma de evaluación de prácticas de laboratorio, que tenga como característica la articulación de la construcción, implementación y presentación de un trabajo experimental y su respectiva evaluación.

\section{Reflexión de las práctica de laboratorio mediante el uso de las uve- heurista}

Dentro de la propuesta que se plantea en este articulo, desde una mirada holística de la educación cientúfica evaluada en las prácticas de laboratorio, no solo debe hacer referencia al lenguaje como habilidad, sino el uso adecuado del mismo al momento de redactar y plasmar la información en contextos cient́ficos, invitando al profesor a enseñar y al estudiante a aprender. Por esto, se desarrolla esta propuesta en el uso de la UVE- HEURÍSTICA y, como estas pueden ser evaluadas por el profesor y el estudiante con respecto a indicadores asociados a contenidos tanto procedimentales, conceptuales y actitudinales. Mediante la técnica de la UVE- heurística, se puede desarrollar para favorecer a estudiantes y profesores, en la comprensión de la naturaleza y los objetivos del trabajo en el laboratorio.

Gowin, (1981) propuso la UVE como un método para ayudar a los estudiantes a comprender la estructura del conocimiento y las formas que tienen los seres humanos de producir este conocimiento. En este sentido, se busca que los estudiantes lleguen con estas capacidades a semestres superiores, ya que posiblemente muchos de ellos, todavía no comprenden la finalidad de una práctica de laboratorio (como estrategia de recolección, análisis e interpretación de datos, que se comunican posteriormente, y que se dan gradias a la experiencia en la práctica) y mucho menos el método de utilización de la UVE - heurística como estrategia metacognitiva. La meta central de la UVE-heurística consiste en mejorar la capacidad de producción textual y síntesis de los docentes y alumnos, gracias al reconocimiento de los criterios como: (problema central, criterio conceptual y metodológico).

\section{Metodologia}

Se desea aplicar esta propuesta tomando un grupo de estudio de primeros semestres de Licenciatura en Química para lograr caracterizar: Inicialmente se mostrara al grupo de estudiantes una situación problema que busca atraer su interés hacia el tema de soluciones, y al mismo tiempo buscar que ellos presenten una propuesta de laboratorio que induya los parámetros (más no condicionamientos) que pueden utilizar para una correcta solución de la misma. Después de presentada la propuesta, desarrollada por ellos y validada, se realizara la práctica de laboratorio, con uno de los procedimientos propuestos por los estudiantes, que mejor se adecue a la correcta solución de la situación. Durante el desarrollo de la práctica se evaluara ciertos "comportamientos" de los estudiantes que previamente fueron categorizados en ítems de la matriz de evaluación (anexo 1), para así tener presente los parámetros principales que permiten un desarrollo exitoso de la práctica y su correcta evaluación. Para la presentación de los resultados de esta práctica, se les pedirá elaborar una UVE-HEURÍSTICA. Para la evaluación de esta UVE-HEURÍSTICA, se tendrá en cuenta una segunda matriz (anexo 2) que contiene una serie de criterios relacionados directamente 
con la disposición y el contenido de la información que se propuso antes de la práctica y los resultados de ésta.

Se realiza esta propuesta para incorporar una nueva forma de evaluación de prácticas de laboratorio que tenga como característica la articulación de la construcción, implementación y presentación de un trabajo experimental y su respectiva evaluación. Dentro de la formación de licenciados en química, la practica experimental se caracteriza por jugar un papel importante en el desarrollo y aplicación de conocimientos impartidos en dases magistrales, que posteriormente se ven reflejadas en un informe de laboratorio, con un contenido que llega a describir mas no explicar ni analizar conocimientos químicos.

Dentro del departamento de química de la Universidad Pedagógica Nacional, es muy común a través de la carrera que las propuestas de prácticas de laboratorio se desarrollen de dos formas:

1. La primera está vinculada con la propuesta explicita del profesor, de lo que se debe realizar en la práctica y lo que se espera obtener de la misma.

2. La segunda se caracteriza porque el estudiante propone un laboratorio de acuerdo a las indicaciones del maestro.

Estas dos formas del desarrollo previo de las prácticas están estrechamente relacionadas con la presentación de un informe de forma teórica, donde se sigue el modelo de descripción más no de análisis de las situaciones, y por lo tanto no hay posterior aplicación a situaciones cotidianas.

Por este motivo la propuesta que aquí se propone, intenta modificar este estilo de prácticas e informes de laboratorio, esperando que los resultados obtenidos sean satisfactorios y significativos en el aprendizaje de la química por parte de los estudiantes universitarios, donde mejoren sus capacidades relacionadas con las competencias científicas, que le permitirán desempeñarse en el campo laboran y social de forma crítica.

\section{Conclusiones}

D El profesor es uno de los principales impulsores de la formación de actitudes hacia la química y su aprendizaje. Por tanto, el desarrollo de estrategias acerca de las prácticas de laboratorio, los métodos de enseñanza y los contenidos científicos pueden influir en las actitudes de las estudiantes, facilitando su desinterés, o por el contrario su interés, relacionado con la formación implícita de un pensamiento reflexivo hacia los conceptos químicos trabajados en la dase.

$>$ Las prácticas son un factor importe en la sociedad, ya que es posible observar en los estudiantes el poco o mucho dominio de los temas vistos a lo largo de su escolaridad dentro de un contexto, lo que demuestra la escasa o alta habilidad intelectual de critica frente a situaciones cotidianas o abstractas que requieren un poco de análisis, aunque en ocasiones, siendo aun más grave, el estudiante no se siente motivado hacia el aprendizaje de las ciencias, siendo allí donde se presenta el decaimiento escolar y posiblemente la deserción de la escuela.

$>$ También cabe resaltar la importancia de generar en los estudiantes una posición investigativa; ya que se suelen presentar las práctica de laboratorio como una receta de cocina, donde no se les resalta que detrás de todas estas, existió en algún momento una investigación, ensayos y errores, y desarrollo epistémico del conocimiento.

$>$ Las prácticas deben ir coordinadas, no solo con dases teóricas, sino con problemas que se propongan en estas dases. Y la evaluación debe ser de todo el proceso como medio y no como un fin. 


\section{Bibliografía}

Acevedo, J.A (1994). La dimensión social de la ciencia y la tecnología. Una perspectiva CTS del desarrollo tecnológico y su evaluación. Ponencia expuesta en las Jomadas de Industrias Químicas: En tomo a treinta años de historia. AIQB y CEP, servicio de publicaciones de la universidad Huelva. 333-329.

Aikenhead, G. (2005). Educación Ciencia Tecnología Sociedad (CTS): Una buena idea como quiera que se le llame. Educación Química. Abril. 114-124.

Aragon, E. (2006). Competencias Científicas Para El Mundo Del Mañana. Gobierno de Aragón. O.C.D.E

Chalmers, A. (1976). ¿Qué es esa cosa llamada ciencia?, España. Editorial Siglo XXI.

Gowin, D.B., (1981). Educating. Comell University Press: Ithaca, NY. 


\section{Anexo 1}

\section{Matriz de evaluacion del laboratorio}

Esta matriz pretende evaluar no solo cuantitativamente, si no también cualitativamente el proceso de desarrollo de la práctica de laboratorio.

\begin{tabular}{|c|c|c|c|c|}
\hline CATEGORY & EXCELENTE & BUENO & ACEPTABLE & REGULAR \\
\hline $\begin{array}{c}\text { Hipótesis } \\
\text { Experimental }\end{array}$ & $\begin{array}{l}\text { La hipótesis } \\
\text { planteada tiene } \\
\text { relación con el } \\
\text { problema, es } \\
\text { coherente, y busca } \\
\text { de una manera } \\
\text { adecuada dar } \\
\text { solución al problema }\end{array}$ & $\begin{array}{l}\text { La hipótesis } \\
\text { planteada tiene } \\
\text { relación con el } \\
\text { problema pero no } \\
\text { da una solución } \\
\text { adecuada. }\end{array}$ & $\begin{array}{l}\text { La hipótesis no } \\
\text { esta relaciona con } \\
\text { el problema, no es } \\
\text { coherente, y no } \\
\text { tiene una solución. }\end{array}$ & $\begin{array}{c}\text { No se propuso una } \\
\text { hipótesis. }\end{array}$ \\
\hline $\begin{array}{c}\text { Diseño } \\
\text { Experimental }\end{array}$ & $\begin{array}{c}\text { El diseño } \\
\text { experimental esta } \\
\text { bien construido y esta } \\
\text { relacionado } \\
\text { directamente con la } \\
\text { hipótesis presentada. }\end{array}$ & $\begin{array}{c}\text { El diseño } \\
\text { experimental bien } \\
\text { construido, pero } \\
\text { la hipótesis deja } \\
\text { muchas preguntas } \\
\text { sin resolver. }\end{array}$ & $\begin{array}{c}\text { El diseño } \\
\text { experimental está } \\
\text { relacionado con la } \\
\text { hipótesis, pero no } \\
\text { es no es una } \\
\text { solución a la } \\
\text { situación } \\
\text { planteada. }\end{array}$ & $\begin{array}{c}\text { El diseño } \\
\text { experimental no está } \\
\text { relacionado a la } \\
\text { hipótesis. }\end{array}$ \\
\hline Materiales & $\begin{array}{c}\text { Todos los materiales } \\
\text { usados en el } \\
\text { experimento son } \\
\text { descritos clara y } \\
\text { precisamente. }\end{array}$ & $\begin{array}{l}\text { La mayoría de los } \\
\text { materiales usados } \\
\text { en el experimento } \\
\text { son descritos } \\
\text { clara y } \\
\text { precisamente. }\end{array}$ & $\begin{array}{l}\text { Casi todos los } \\
\text { materiales usados } \\
\text { en el experimento } \\
\text { están descritos. }\end{array}$ & $\begin{array}{l}\text { No se presentan } \\
\text { materiales y no hay } \\
\text { descripción del } \\
\text { proceso en un } \\
\text { diagrama de fiujo. }\end{array}$ \\
\hline Datos & $\begin{array}{l}\text { Los datos son } \\
\text { presentados de una } \\
\text { manera precisa y } \\
\text { ordenada en tablas } \\
\text { y/o gráficas. Estas } \\
\text { debidamente } \\
\text { marcadas y/o } \\
\text { enumeradas. }\end{array}$ & $\begin{array}{c}\text { Los datos son } \\
\text { presentados de } \\
\text { una manera } \\
\text { precisa y } \\
\text { ordenada en } \\
\text { tablas y/o } \\
\text { gráficas. Pero } \\
\text { estas no están } \\
\text { debidamente } \\
\text { marcadas y/o } \\
\text { enumeradas. }\end{array}$ & $\begin{array}{c}\text { Una } \\
\text { representación } \\
\text { precisa de los } \\
\text { datos en forma } \\
\text { escrita. }\end{array}$ & $\begin{array}{l}\text { Los datos no son } \\
\text { mostrados. }\end{array}$ \\
\hline Cálculos & $\begin{array}{l}\text { Se muestra todos los } \\
\text { cálculos y los } \\
\text { resultados son } \\
\text { correctos. }\end{array}$ & $\begin{array}{c}\text { Se muestra } \\
\text { algunos cálculos y } \\
\text { los resultados son } \\
\text { correctos. }\end{array}$ & $\begin{array}{l}\text { Se muestra } \\
\text { algunos cálculos } \\
\text { pero estos son } \\
\text { incorrectos. }\end{array}$ & $\begin{array}{l}\text { No se muestra } \\
\text { ningún cálculo. }\end{array}$ \\
\hline Seguridad & $\begin{array}{l}\text { El laboratorio es } \\
\text { llevado a cabo con } \\
\text { toda atención a los } \\
\text { procedimientos de } \\
\text { seguridad. Y se } \\
\text { portan todos los } \\
\text { implementos de } \\
\text { seguridad } \\
\text { personal(bata, } \\
\text { guantes, gafas) }\end{array}$ & $\begin{array}{l}\text { El laboratorio es } \\
\text { llevado a cabo } \\
\text { con poca } \\
\text { atención, a los } \\
\text { procedimientos de } \\
\text { seguridad. Y se } \\
\text { portan todos los } \\
\text { implementos de } \\
\text { seguridad } \\
\text { personal(bata, } \\
\text { guantes, gafas) }\end{array}$ & $\begin{array}{l}\text { El laboratorio es } \\
\text { llevado a cabo con } \\
\text { algo de atención a } \\
\text { los pocos } \\
\text { procedimientos de } \\
\text { seguridad. Y no se } \\
\text { portan todos los } \\
\text { implementos de } \\
\text { seguridad } \\
\text { personal(bata, } \\
\text { guantes, gafas) }\end{array}$ & $\begin{array}{l}\text { Los procedimientos } \\
\text { de seguridad fueron } \\
\text { ignorados y/o } \\
\text { algunos aspectos del } \\
\text { experimento plantean } \\
\text { un riesgo para la } \\
\text { seguridad del } \\
\text { estudiante o de otros } \\
\text { individuos. Y no } \\
\text { portan todos los } \\
\text { implementos de } \\
\text { seguridad personal. }\end{array}$ \\
\hline
\end{tabular}


Anexo 2

Matriz de evaluación informe de laboratorio (v heurística)

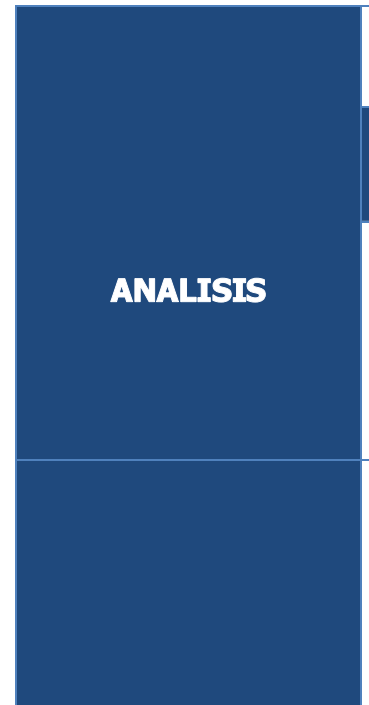

CONCLUSIONES

HIPÓTESTS

CONCEPTOS CLAVE

DISEÑO

EXPERTMENTAL

\section{Analiza e interpreta los
resultadosivasean:
valores, Gráficas o}

tabulaciones,

contestando

claramente, la pregunta

Equmblerfan slarictadide

problema o pregunta

que se quiere

responder. Abarca el

Arecteaniterfosqto su

expeiphastatice los

mectidpendebntexto

equimento,

relacionándolos, con la

hipótesis, los conceptos

y el análisis, buscando

sintetizar el trabajo en

el laboratorio y los

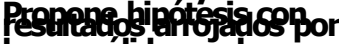

gases sólidas en los

conocimientos que se

tienen del problema a

resolver o la pregunta a

responder.

Comprende y utiliza

conceptos, principios,

leyes y teorías

fundamentales que

tienen relación con la

solución al problema 0

respuesta a la pregunta.

Propone un diseño

experimental que

induye la medición y el

control de variables

presentes en el

problema que se quiere

resolver.

Describe daramente

fenómenos de interés aprendidos mediante conceptos y registros de datos: sucesos, objetos,

entre otros
El análisis 0

interpretación de

los resultados no

sobafinestajão

folvaón BLE

lo tanto no es

adecuada para

solucionar el

paplamatadon del

BFeglera o la

Blagtondaque se

quiere responder

Axglitiepstetegde

Eifoepisforta

los resultados de

la experiencia,

pero no relaciona

todos los pasos

realizados en la

Prepcere dipótesis,

Parorarofínde los

conocimientos que

se tienen dé

problema a

resolver o la

pregunta a

responder.

Comprende y

utiliza

parcialmente

conceptos,

principios, leyes y

teorías

fundamentales

que tienen

relación con la

solución al

problema 0

respuesta a la

pregunta.

Propone un diseño

experimental que

induye la

medición y el

control de

variables para la

solución del

problema que se

quiere resolver.

Describe de forma

superficial los

fenómenos

mediante el

registro de datos:

sucesos, objetos,

entre otros.
El análisis 0

interpretación
de loS MAL
resuttaddof, HO

PJtétara la análisis

forrmuacton lael

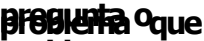

Bxqlalitepa

Plaptopada

Teniendo en

cuenta criterios

que abarcan lo

procedimental,

rocapgentería,

Gino tezcrica

yucediltoxto

Euifante la

Brepore

hipótesis sin

tener en cuenta

los

conocimientos No presenta

que se tiene del hipótesis

problema a

resolver o la

pregunta a

responder.

No Comprende

los conceptos,

principios, leyes

y teorías

fundamentales y

por lo tanto no

los utiliza en la

solución al

problema 0

respuesta a la

pregunta.

No se formula

pregunta

condusiones

No entiende los

conceptos ni

principios.

Propone un

diseño

experimental

que no induye

la medición ni el

control de

variables.

Describe y

registra

acontecimientos,

que no están

no presenta

relacionados con resultados

lo sucedido

durante-la

práctica.

no presenta

diseño

experimental

Reiaciones Enseñanza Âprendizaje 
